ARTICLE

DOI: $10.1038 / s 41467-017-00165-8$

\title{
Slow conformational exchange and overall rocking motion in ubiquitin protein crystals
}

Vilius Kurauskas ${ }^{1,2,3}$, Sergei A. Izmailov4, Olga N. Rogacheva ${ }^{4}$, Audrey Hessel ${ }^{1,2,3}$, Isabel Ayala ${ }^{1,2,3}$, Joyce Woodhouse ${ }^{1,2,3}$, Anastasya Shilova ${ }^{5}$, Yi Xue ${ }^{6}$, Tairan Yuwen ${ }^{6}$, Nicolas Coquelle $1,2,3$, Jacques-Philippe Colletier (iD 1,2,3, Nikolai R. Skrynnikov (i) ${ }^{4,6}$ \& Paul Schanda (i) 1,2,3

Proteins perform their functions in solution but their structures are most frequently studied inside crystals. Here we probe how the crystal packing alters microsecond dynamics, using solid-state NMR measurements and multi-microsecond MD simulations of different crystal forms of ubiquitin. In particular, near-rotary-resonance relaxation dispersion (NERRD) experiments probe angular backbone motion, while Bloch-McConnell relaxation dispersion data report on fluctuations of the local electronic environment. These experiments and simulations reveal that the packing of the protein can significantly alter the thermodynamics and kinetics of local conformational exchange. Moreover, we report small-amplitude reorientational motion of protein molecules in the crystal lattice with an $\sim 3-5^{\circ}$ amplitude on a tens-of-microseconds time scale in one of the crystals, but not in others. An intriguing possibility arises that overall motion is to some extent coupled to local dynamics. Our study highlights the importance of considering the packing when analyzing dynamics of crystalline proteins.

\footnotetext{
${ }^{1}$ Université Grenoble Alpes, 71 avenue des martyrs, F-38044 Grenoble, France. ${ }^{2}$ CEA, Institut de Biologie Structurale, 71 avenue des martyrs, F-38044 Grenoble, France. ${ }^{3}$ CNRS, Institut de Biologie Structurale, 71 avenue des martyrs, F-38044 Grenoble, France. ${ }^{4}$ Laboratory of Biomolecular NMR, St. Petersburg State University, St. Petersburg, 199034, Russia. ${ }^{5}$ European Synchrotron Radiation Facility, 71 avenue des martyrs, F-38044 Grenoble, France. ${ }^{6}$ Department of Chemistry, Purdue University, 560 Oval Drive, West Lafayette, IN 47907-2084, USA. Vilius Kurauskas, Sergei A. Izmailov, and Olga N. Rogacheva authors contributed equally to this work. Correspondence and requests for materials should be addressed to N.R.S. (email: nikolai@purdue.edu) or to P.S. (email: paul.schanda@ibs.fr)
} 
$\mathrm{P}$ roteins perform their functions in aqueous solution at ambient temperatures by sampling a multitude of conformational states involved, for example, in enzymatic turnover or binding to different partners. Understanding protein function requires characterizing the three-dimensional structures, ideally of all these thermally accessible conformations, and deciphering how the different conformations interconvert. The structural basis for understanding protein function have been laid primarily through X-ray diffraction (XRD) of proteins embedded in crystals. Recent methodological advances in XRD aim at characterizing also the structural heterogeneity by fitting multiple conformers-rather than a single structure-into the electron density obtained from Bragg diffraction peaks, or by additionally making use of diffuse scattering intensity ${ }^{1-6}$. The direct structural insight into protein dynamics that one may obtain from such ensemble crystallographic approaches may shed light on molecular mechanisms of functional processes; in particular, when combined with techniques that provide access to kinetics and thermodynamics, such as solution-state NMR spectroscopy ${ }^{7}$, one may obtain a fairly complete spatio-temporal picture, which may ultimately lead to a more detailed understanding of protein function.

The densely packed environment of crystals differs substantially from solution, raising the question how packing may alter the properties of proteins. Structures obtained from crystalline proteins are generally in excellent agreement with state-ofthe-art solution-NMR structures, and are generally as good in predicting solution-NMR structural parameters as the NMR structures themselves $^{8,9}$. However, whether dynamics, i.e., the ensemble of co-existing conformers and their pairwise interconversion rates, are also conserved in crystals is less well studied. Evidence that the dynamics required for function are retained in crystals, at least in some cases, comes from the observation of functional activity, such as ligand binding and enzymatic catalysis, in crystals ${ }^{10-14}$. Direct experimental investigations of the dynamics of proteins in crystals at the atomic level have turned out to be difficult. Early studies focused on comparing XRD temperature factors (B-factors) with solution-state NMR order parameters $\left(S^{2}\right)^{15-17}$. Such comparisons only had limited success and remained ambiguous, because of the very different nature and information content of these observables (see ref. ${ }^{18}$ and references therein). Recent developments in magic-angle spinning solid-state NMR (MAS ssNMR) of proteins have allowed a more direct comparison of NMR parameters in solution and crystals $^{19-21}$. Furthermore, comparative molecular dynamics (MD) simulations of proteins embedded in an explicit crystal lattice and in solution also produced useful insights, at least over the time scales accessible to MD (typically sub-microsecond) ${ }^{22-25}$. The picture arising from these NMR and MD studies suggests that the crystal lattice has indeed only little impact on dynamics on sub-microsecond time scales: changes are primarily found in loops engaged in crystal contacts, whereas secondary structure elements and loops remote from neighboring molecules showed similar order parameters. Many biological processes, however, occur on longer time scales, often micro-to-milliseconds ( $\mu s-\mathrm{ms})$, and they may involve rare excursions to low-populated "minor states". In many cases, such rare conformations seem to be crucial for functional activity ${ }^{26}$, and they may involve larger-scale rearrangements affecting a bigger portion of the structure. Whether such slow dynamic processes are sustained in crystals, and how the packing alters these motions is poorly understood. MD simulation methods only recently attained the ability to model those time scales, and experimental NMR methods likewise became available only recently.

Here we use a combination of ssNMR experimental methods with microsecond-long MD simulations of explicit crystal lattices, in order to investigate how the crystalline environment impacts slow dynamics of the $8.6 \mathrm{kDa}$ regulatory protein ubiquitin. The dynamics of this protein in solution have been extensively characterized experimentally and computationally. In particular, microsecond motion has been shown for the loop connecting ubiquitin's $\beta 4$ strand and the $3_{10}$ helix, comprising residues E51 to R54, which exists in two distinct states that differ by a flip of the D52/G53 peptide plane, along with an alteration of hydrogen bonds within this $\beta$-turn and to the adjacent helix ${ }^{27-32}$. These two states correspond to so-called type-I and type-II $\beta$-turns, respectively, herein referred to as $\beta$ I and $\beta$ II states. The dynamic exchange process between these two conformations has been proposed to result in small (within $1 \AA$ ) but discernible reshaping of the entire molecule ${ }^{30}$, and perturbation of the $\beta$-turn equilibrium has been shown to allosterically alter the binding of proteins to a surface located on the opposite side of the ubiquitin molecule $e^{30}$. This dynamic process is thus thought to be of considerable functional relevance for ubiquitin. Different crystal structures are available that are in either of these two states, and millisecond-long MD simulations in solution have detected the corresponding microsecond exchange process $^{32}$. Previous ssNMR data revealed that this $\mu$ s dynamics is present in one of ubiquitin's crystal forms ${ }^{33,34}$.

In addition to this internal motional process, we have recently provided evidence that ubiquitin molecules undergo overall "rocking" motion in the crystal ${ }^{35}$. This motion was detected by a comparative ssNMR $R_{1 \rho}$ relaxation measurement of different crystal forms, which revealed that in one of the crystal forms, termed cubic-PEG-ub, elevated relaxation rates throughout the molecule can be explained by an overall reorientational motion with an amplitude of several degrees. The presence of this motion was also confirmed by MD simulations. It was furthermore shown that the observed rocking has direct implications for X-ray crystallography: the crystal form that features rocking motion gives rise to low-resolution diffraction data and high Wilson B-factors in crystallographic experiments at $100 \mathrm{~K}$. However, our previous study did not provide a precise estimate of the rocking motion time scale, and only a range from $\sim 100$ ns to $100 \mu$ s was indicated ${ }^{35}$.

Here we investigate in parallel two different crystal forms, thus avoiding any bias that would arise from comparing dynamics measured by different methods (i.e., solution and solid-state methods). We use Bloch-McConnell-type $R_{1 \rho}$ relaxation dispersion experiments, which were recently introduced to solid-state $\mathrm{NMR}^{34}$, to probe on a per-residue basis the dynamics-induced modulation of the chemical shift of ${ }^{15} \mathrm{~N}$ nuclei. In addition, we use $R_{1 \rho}$ experiments at higher radio-frequency field strengths, near the rotary-resonance condition $\omega_{\mathrm{RF}}^{\text {eff }}=\omega_{\mathrm{MAS}}{ }^{34,36,37}$, which we refer to here as near-rotary-resonance relaxation dispersion (NERRD) experiment. This experiment is sensitive to the angular fluctuations of the $\mathrm{H}-\mathrm{N}$ bond vectors. A systematic analysis of experimental NERRD data against numerical simulations provides information about the angular motional amplitude of $\mu \mathrm{s}$ motions.

The combined experimental data establish that crystal contacts indeed alter the kinetics (exchange rates) and thermodynamics (relative populations of states) of $\mu$ s motions. We find that the relative populations of the exchanging states, $\beta \mathrm{I}$ and $\beta \mathrm{II}$, can be inverted in going from one crystal to another, and that in both analyzed crystal forms the exchange rates are slowed down significantly with respect to solution. Multi- $\mu$ s long MD trajectories of WT and mutant proteins confirm these experimental findings, and identify the molecular origins of these altered dynamics. Using NERRD data, we establish that overall rocking occurs on the tens-of-microseconds time scale, with an amplitude of about $3-5^{\circ}$. The crystal form that is amenable to rocking also features 

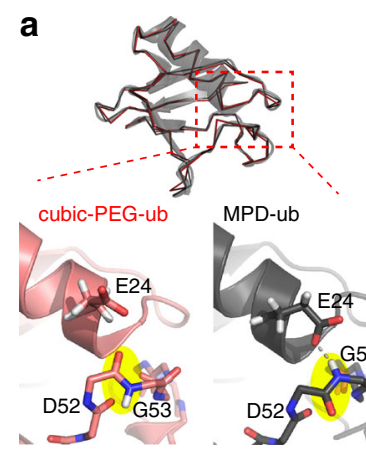

Type-I $\beta$-turn

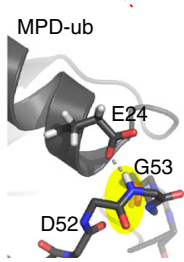

Type-II $\beta$-turn b

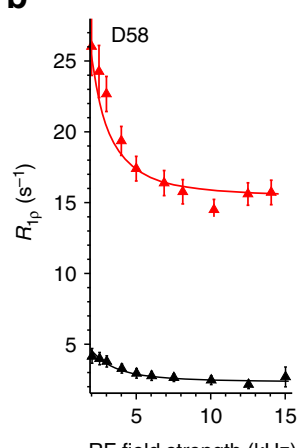

C

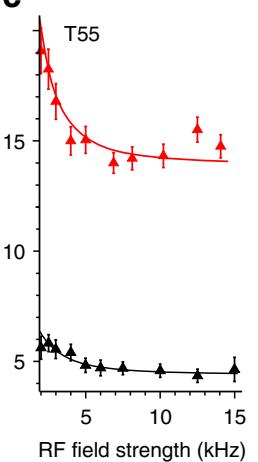

d

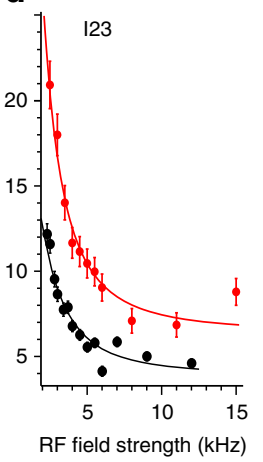

e

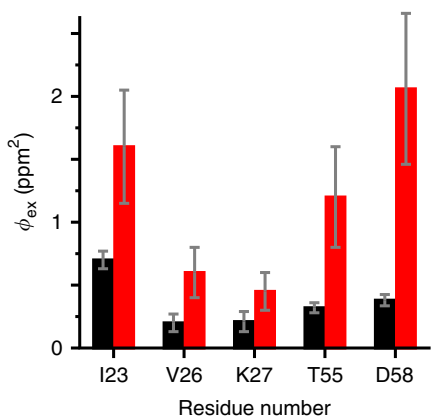

Fig. 1 Evidence for $\beta$-turn exchange in MPD-ub (black) and cubic-PEG-ub (red) crystals. a Structures of ubiquitin in the two crystal forms. Top: superposition of the main chains in the two crystals, shown as $C^{\alpha}$ backbone traces; for cubic-PEG-ub only one of the two non-equivalent chains is shown. For clarity, the semi-transparent cartoon representation is shown only for MPD-ub. Bottom: zoom into the $\beta$-turn region. The peptide bond undergoing a flip is highlighted in yellow. b-d BMCRD profiles of ${ }^{15} \mathrm{~N}$ sites in the two ubiquitin crystal forms, obtained at a sample temperature of $300 \mathrm{~K}$. Data shown here for D58 and T55 were obtained at $B_{0}$ field strengths corresponding to ${ }^{1} \mathrm{H}$ Larmor frequencies of $600 \mathrm{MHz}$, and those of $\mathrm{I} 23$ at $950 \mathrm{MHz}$, respectively. Error bars of $R_{1}$, values were obtained by standard Monte Carlo analyses, described in the Methods section. Solid lines correspond to a global two-site exchange model fit of data from residues 123, V26, K27, T55, D58 within each of the crystal forms (solid lines). The fits were performed separately for each of the two crystal forms using in each case the same cluster of the aforementioned five residues. Note that the resonances of E24 and N25 are not visible, presumably as a consequence of the conformational exchange. The comprehensive set of BMCRD data and fitting results can be found in Supplementary Figs. 2, 3, and 5. e $\phi_{\mathrm{ex}}$ values, $\phi_{\mathrm{ex}}=p_{\mathrm{A}} p_{\mathrm{B}}(2 \pi \Delta \delta)^{2}$ in cubic-PEG-ub (red) and MPD-ub (black), obtained from the joint fit of residues I23, V26, K27, T55, and D58, exemplified by curves in $\mathbf{b}-\mathbf{d}$. Here $p_{\mathrm{A}}$ and $p_{\mathrm{B}}$ are the populations of the two conformers, while $\Delta \delta$ is the chemical shift difference expressed in the units of ppm. Fitted exchange time constants are reported in Table 1

extensive $\beta \mathrm{I} / \beta \mathrm{II}$ exchange. Furthermore, both rocking and exchange dynamics occur on a similar time scale, suggesting some degree of cooperativity between local and overall motions. Although our attempts to capture this effect by means of the rationally designed mutations proved unsuccessful, we believe that this scenario remains a possibility in this and other protein crystals. Our results highlight the importance of crystal packing effects in the context of crystallography-based investigation of protein dynamics.

\section{Results}

Theory. In order to probe $\mu$ s dynamics we employ ssNMR ${ }^{15} \mathrm{~N}$ $R_{1 \rho}$ relaxation dispersion (RD) experiments. RD experiments measure the transverse magnetization decay rate constant, $R_{1 \rho}$, under an applied radio-frequency field for each of the ${ }^{15} \mathrm{~N}$ backbone spins across the protein, and investigate how this decay rate constant depends on the field-strength, $\nu_{\mathrm{RF}}$, of the applied RF field. In MAS ssNMR, two different physical mechanisms can give rise to (non-flat) RD profiles:

(i) If the exchanging states differ in their local electronic environment around a given ${ }^{15} \mathrm{~N}$ nucleus, and if the exchange process occurs on a $\mu \mathrm{s}-\mathrm{ms}$ time scale, the corresponding chemical-shift modulation contributes to the $R_{1 \rho}$ decay rate constant. This enhancement of $R_{1 \rho}$ can be scaled down by applying a sufficiently strong RF field, i.e., $R_{1 \rho}$ decreases with increasing $\nu_{\mathrm{RF}}$. This effect, known as "Bloch-McConnell RD" in solution-state $\mathrm{NMR}^{38}$, depends on the chemical shift difference between the exchanging sites, as well as the time scale of the exchange, the relative populations of the exchanging states and the amplitude of the RF field.

(ii) Fluctuation of the dipolar and CSA interactions relative to the reference frame of the crystallite also induces enhanced $R_{1 \rho}$ relaxation, in addition to the above effect that fluctuation of the isotropic chemical shift has ${ }^{34,} 37$. If the relevant motions are in the $\mu \mathrm{s}-\mathrm{ms}$ range then this effect can be scaled down not only by application of the RF field, but also by magic angle sample spinning. Mathematically this is expressed by the formula derived by Kurbanov et al. ${ }^{39}$ which (in the case of sufficiently strong RF field surpassing the RF carrier offset $\omega_{\mathrm{RF}}^{\text {eff }} \gg \Omega$ ) can be rendered in the following familiar form:

$$
\begin{array}{r}
R_{1 \rho}=\frac{1}{8} D_{\mathrm{NH}}^{2}\left[4 j_{0}+J\left(\omega_{\mathrm{N}}-\omega_{\mathrm{H}}\right)+3 J\left(\omega_{\mathrm{N}}\right)+6 J\left(\omega_{\mathrm{H}}\right)\right. \\
\left.+6 J\left(\omega_{\mathrm{N}}+\omega_{\mathrm{H}}\right)\right]+\frac{1}{18} C_{\mathrm{N}}^{2}\left[4 j_{0}+3 J\left(\omega_{\mathrm{N}}\right)\right]
\end{array}
$$

$$
\begin{aligned}
j_{0}= & \frac{1}{6}\left[J\left(\omega_{\mathrm{RF}}-2 \omega_{\mathrm{MAS}}\right)+2 J\left(\omega_{\mathrm{RF}}-\omega_{\mathrm{MAS}}\right)\right. \\
& \left.+2 J\left(\omega_{\mathrm{RF}}+\omega_{\mathrm{MAS}}\right)+J\left(\omega_{\mathrm{RF}}+2 \omega_{\mathrm{MAS}}\right)\right] .
\end{aligned}
$$

Here $D_{\mathrm{NH}}=-\left(\mu_{0} / 4 \pi\right)\left(\hbar \gamma_{\mathrm{N}} \gamma_{\mathrm{H}} / r_{\mathrm{NH}}^{3}\right)$ is the dipolar coupling strength, $C_{\mathrm{N}}=\omega_{\mathrm{N}}\left(\sigma_{\perp}^{\mathrm{N}}-\sigma_{\|}^{\mathrm{N}}\right)$ is the anisotropy of the chemical-shift tensor, $\omega_{\mathrm{RF}}=2 \pi \nu_{\mathrm{RF}}$ is the spin-lock field strength, and $\omega_{\text {MAS }}=2 \pi \nu_{\text {MAS }}$. The explicit form of spectral densities $J(\omega)$ depends on the adopted model of motion; the empirical Lipari-Szabo-style expression is $J(\omega)=\frac{2}{5}\left(1-S^{2}\right) \tau_{c} /\left(1+\omega^{2} \tau_{c}^{2}\right)$, where $S^{2}$ is the generalized order parameter, expressing the motional amplitude of the bond vector, and $\tau_{\mathrm{c}}$ is the correlation time of the motion.

Of particular importance in Eqs. (1) and (2) are spectral densities $J\left(\omega_{\mathrm{RF}} \pm n \omega_{\mathrm{MAS}}\right)(n=1,2)$. The meaning of these terms is as follows: if the motional process is sufficiently slow, $\omega_{\mathrm{RF}} \tau_{c} \geq 1$ or $\omega_{\text {MAs }} \tau_{c} \geq 1$, then relaxation can be partially suppressed due to refocusing of the dipolar interaction by RF field or MAS, respectively. However, it may also happen that refocusing due to RF field and due to MAS in part cancel each other out (reminiscent of dipolar recoupling). This phenomenon occurs near rotary-resonance conditions, $\omega_{\mathrm{RF}}=n \omega_{\mathrm{MAS}}$ or, equivalently $\nu_{\mathrm{RF}}=n \nu_{\mathrm{MAS}}(n=1,2)$, and is accompanied by increase in the $R_{1 \rho}$ decay rate constant. Such behavior (illustrated by means of numerical simulations, Supplementary Fig. 1) is the basis of NERRD experiment. We note NERRD-type experiments have been used in previous studies ${ }^{40-44}$. 
The observation of an increase of $R_{1 \rho}$ when approaching the rotary-resonance condition is an unambiguous signature of $\mu \mathrm{s}$ motions. Assuming that dynamics can be modeled as an exchange process, the exact shape of such NERRD profiles depends on the time scale of motion, the relative populations of the involved states, as well as the difference in orientation of the $\mathrm{NH}$ bond vector between the exchanging states ${ }^{36,37}$. NERRD thus provides insight into the angular amplitude of the motion, and complements Bloch-McConnell RD data, which probe motions by their isotropic chemical-shift modulation. Importantly, in solutionstate NMR this type of information is unavailable, because the rapid (ns) molecular tumbling averages the anisotropic interactions such that they are not informative of slow dynamics.

Both of the above relaxation-dispersion mechanisms, (i) and (ii), simultaneously lead to RF-field-dependent $R_{1 \rho}$ decay rate profiles whenever $\mu \mathrm{s}-\mathrm{ms}$ motions are present. However, experimental parameters can be chosen such as to separate the two effects. Provided that a fairly large sample-spinning frequency $\nu_{\text {MAS }}$ is used, the $R_{1 \rho} \mathrm{RD}$ profiles in the low RF-field range, typically $\nu_{\mathrm{RF}} \leq 10 \mathrm{kHz}$, are dominated by isotropic chemical-shift mechanism. For simplicity, we employ the term "Bloch-McConnell relaxation dispersion" (BMCRD) for this situation. On the other hand, in the vicinity of the rotaryresonance conditions, which in our NERRD experiments is at $\nu_{\mathrm{RF}}=\nu_{\mathrm{MAS}}=44 \mathrm{kHz}$ and thus well separated from the BMCRD regime, it is the angular fluctuation of the $\mathrm{NH}$ bond that dominates the RD profiles ${ }^{34,37}$. For simplicity, we will reserve the term NERRD for the situation where relaxation due to modulation of isotropic chemical shift is fully suppressed by the strong RF field and the near-rotary-resonance dispersion effect arises solely from the anisotropic interactions.

We note, however, that the two described relaxation dispersion phenomena (BMCRD, NERRD) are not fundamentally different but rather reflect two mechanisms of line broadening induced by $\mu$ s motion. The two respective data sets can be fitted jointly or independently, and such fits can in ideal cases provide exchange rates, population levels of involved states, chemical-shift differences and jump angles. In systems where the exchange process is fast (less than $\sim 100 \mu \mathrm{s}$ ), which is the case here, it is not possible to disentangle populations and chemical-shift changes, and only their product can be obtained; likewise, populations and jump angles also become entangled (see further discussion below and Supplementary Fig. 4).

Crystal contacts slow down type-I/type-II $\beta$-turn exchange. In this study, we investigate dynamics in two different crystal forms of ubiquitin that are obtained in the presence of two different precipitation agents (2-methyl-2,4-pentanediol (MPD) and polyethylene glycol (PEG), see Methods) and are referred to as MPD-ub and cubic-PEG-ub, respectively. XRD-based crystal structures of these different forms have been previously solved at cryo-temperatures $(100 \mathrm{~K})^{35,45,46}$. While these X-ray data reveal that ubiquitin adopts essentially the same overall structure (backbone RMSD of $0.5 \AA$ ), they differ in the conformation of the loop region encompassing residues E51 to R54: in MPD-ub this region forms a so-called type-II $\beta$-turn, while it is in $\beta \mathrm{I}$ conformation in cubic-PEG-ub (Fig. 1a). Note that the predominant state in solution is $\beta \mathrm{I}^{35,45-47}$ with an estimated $>95 \%$ population, in exchange with an alternate state which is believed to be $\beta \mathrm{II}^{30}$.

Figure 1b-d shows Bloch-McConnell RD data for three representative amide sites in MPD-ub and cubic-PEG-ub. The non-flat RD profiles for residues located in the $\beta$-turn region and the adjacent $\mathrm{N}$-terminal part of the $\alpha$-helix unambiguously show that this part of the molecule undergoes $\mu$ s dynamics in both crystals. A joint fit of a two-state exchange model to data from the five residues located in this region is shown as solid lines (see Methods). The use of a two-state exchange model appears justified in the light of the two distinct states observed in different crystal structures and MD simulations (ref. ${ }^{32}$ and MD data below). The exchange time constant obtained from these fits is $\tau_{\mathrm{ex}}=90 \pm 25 \mu \mathrm{s}$ for MPD-ub and $125 \pm 45 \mu$ s for cubic-PEG-ub. We want to stress the fact that the absolute "plateau" levels of these curves are significantly higher in cubic-PEG-ub than in MPD-ub throughout the molecule, as can be readily seen in the three examples in Fig. 1b-d. While the plateau is not relevant for fits of BMCRD data, this finding of elevated $R_{1 \rho}$ rates points to the presence of overall angular motion of ubiquitin molecules in cubic-PEG-ub, as we will outline below.

In order to gain insight into the angular motions of the $\mathrm{NH}$ bond vectors, we measured NERRD profiles. Figure 2 shows the largest NERRD effects observed in MPD-ub, which are found for the amide sites of D52 and R54, which are adjacent to the peptide plane D52/G53 that undergoes a ca. $180^{\circ}$ flip when exchanging between $\beta \mathrm{I}$ and $\beta \mathrm{II}$ conformations. (The amide signal of G53 is not resolved in the spectra of MPD-ub.) Other residues do not show significantly rising NERRD profiles, as exemplified by the data of three residues depicted in black in Fig. 3. An independent experiment at a different MAS frequency $(20 \mathrm{vs} .44 \mathrm{kHz}$ in the current experiment) also exhibited the largest NERRD effect for
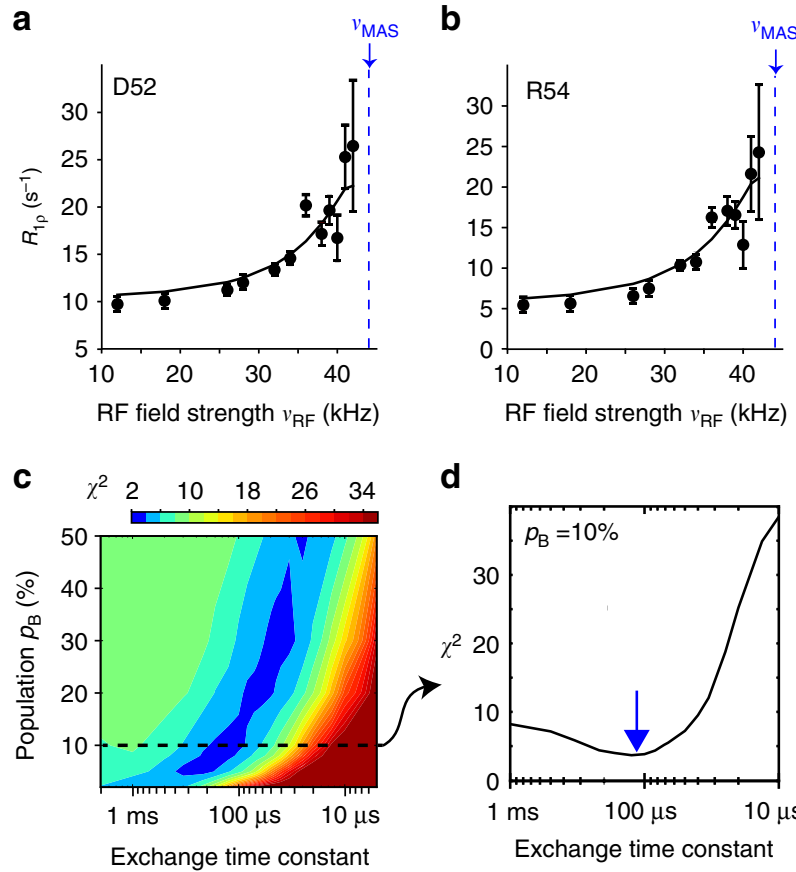

d

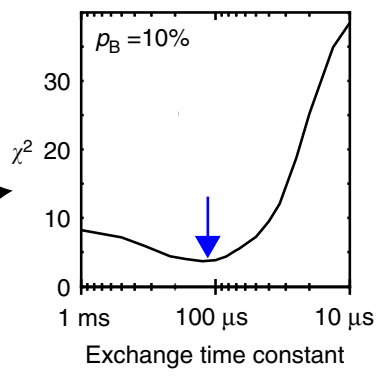

Fig. 2 Angular motion in the $\beta$-turn of MPD-ub crystals probed by NERRD NMR. $\mathbf{a}, \mathbf{b}$ NERRD dispersion data of the two amide sites in MPD-ub that are adjacent to the flipping peptide plane D52/G53, recorded at 44.053 $\mathrm{kHz}$ MAS frequency. Error bars of $R_{1}$ values were obtained by standard Monte Carlo analyses, described in the Methods section. Solid lines show fit curves from a two-site jump model that assumes that the minor conformation has a population of $p_{\mathrm{B}}=10 \%$. c $\chi^{2}$ surface of a grid-search of simulated NERRD curves against the experimental data in $\mathbf{a}, \mathbf{b}$. Hereby, a common minor-state population $p_{B}$ and exchange correlation time $\tau_{\mathrm{ex}}=1 /\left(k_{\mathrm{AB}}+k_{\mathrm{BA}}\right)$ were assumed for the two residues, and the jump angle was separately fitted for each residue. More details of this NERRD fit are reported in Supplementary Fig. 4. d Cross-section across the $\chi^{2}$ surface along the $\tau_{\text {ex }}$ dimension corresponding to a minor-state population of $10 \%$. This minor-state population level is suggested by analyses of BMCRD data, as explained in the text. The best-fit exchange time constant with this assumption is $\tau_{\mathrm{ex}} \sim 100 \mu \mathrm{s}$ 
a
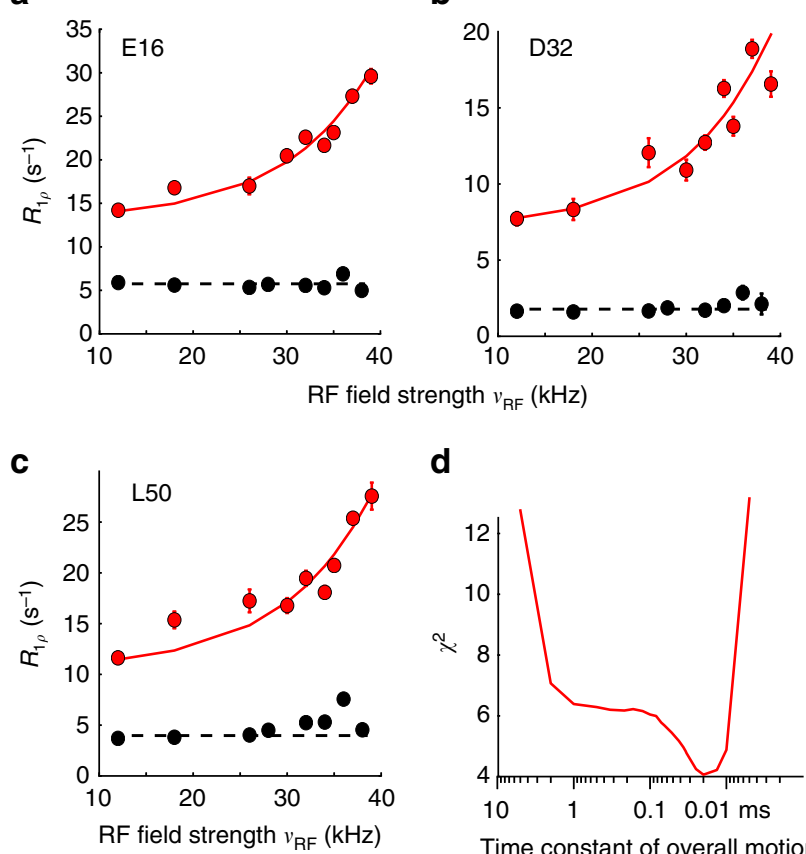

d

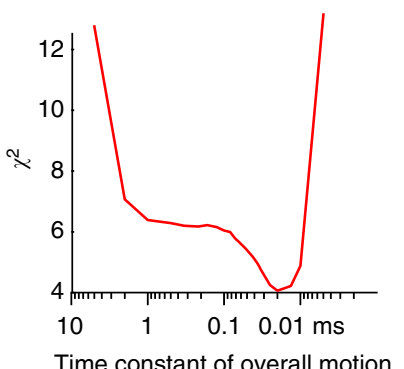

Fig. 3 Evidence for rocking motion in cubic-PEG-ub crystals from NERRD NMR measurements. a-c NERRD data of representative amide sites outside the dynamic $\beta$-turn region for MPD-ub (black) and cubic-PEG-ub (red). Error bars were obtained from Monte Carlo analysis, described in the Methods section. The solid red lines correspond to a two-state fit of data from 22 amide sites with a common exchange time constant and perresidue-adjustable motional amplitude (all shown in Supplementary Fig. 6). d $\chi^{2}$ value of this fit as a function of the jump time constant. In fitting these NERRD data, we have assumed that protein rocking can be approximated as two-site jump process, which is a crude but justifiable model ${ }^{70}$

residues D52 and R54 in MPD-ub ${ }^{35}$, whereas all other residues showed either small NERRD profiles (residues I23, E51, T55) or no significant NERRD effect, mirroring the present data.

To quantitatively rationalize these data, we used numerical spin-dynamics simulations to obtain theoretical NERRD profiles over a grid of exchange parameters, $p_{\mathrm{B}}$ and $\tau_{\mathrm{ex}}$, and fitted the experimental data against this grid of simulated curves. Solid lines in Fig 2a, b show the best-fit curves for such a joint fit of data from D52 and R54, assuming that they have one common excited-state population and exchange time constant. Figure $2 \mathrm{c}$ shows the corresponding plot of the $\chi^{2}$ surface. Although the population levels obtained from these fits have significant uncertainty, the time scale converges to $\sim 50-200 \mu \mathrm{s}$. This is in excellent agreement with the independent Bloch-McConnell $\mathrm{RD}$ data, as illustrated in Fig. 1b-d. In addition to confirming the time scale of the process, NERRD data provide information about the amplitude of the NH motion of D52 and R54, which is found to be on the order of $7-11^{\circ}$, depending on the assumed populations (Supplementary Fig. 4). Interestingly, this angle is similar to the difference between the orientations that these two bonds assume in $\beta \mathrm{I}$ and $\beta \mathrm{II}$ states, which is 12 and $14^{\circ}$ for the two respective residues (comparing PDB entries $1 \mathrm{UBQ}$ and 3ONS).

Taken together, both Bloch-McConnell RD and NERRD data show that the $\beta$-turn dynamics in MPD-ub occurs on a time scale of ca. $100 \mu \mathrm{s}$; this exchange process causes chemical shift modulation in the $\beta$-turn region (Fig. 1) and angular jumps of the amides of D52 and R54, as revealed by NERRD (Fig. 2).

It is interesting to note that the two crystals, which have very different packing environment around the exchanging region, have similar exchange kinetics, but that these kinetics are rather different to the case of solution, where the exchange process has a time constant of $\tau_{\mathrm{ex}}=6 \pm 2 \mu \mathrm{s}$ at the same temperature, i.e., is about 15 times faster $^{30}$. The significant slowdown of the exchange process in the crystals is not due to the increased viscosity induced by the precipitation agent: it has been shown ${ }^{33}$ that even in the presence of MPD concentrations as high as $45 \%$ in solution all CPMG RD profiles were flat, i.e., the underlying process was too fast to induce $\mathrm{RD}$, whereas in MPD-ub crystals very large CPMG RDs were detected, thus confirming that MPD is not the principal cause for slowing of the exchange dynamics ${ }^{33}$. As noted above, the "ground state" is different between the two crystals, being $\beta \mathrm{I}$ in cubic-PEG-ub (as well as in solution), and $\beta \mathrm{II}$ in MPD-ub. Our findings show that the nature of the ground state does not dictate the kinetics, because in solution and cubic-PEG$\mathrm{ub}$, which have both $\beta \mathrm{I}$ as major state, the kinetics are very different.

$\boldsymbol{\beta} \mathbf{I} / \mathbf{\beta I I}$ populations differ in the different crystals. Inspection of the BMCRD data in Fig. 1 reveals a noticeable difference between the two crystals: the amplitude of these RD profiles for residues in cubic-PEG-ub is considerably larger than those of their counterparts in MPD-ub, on average by a factor $3.3 \pm 1.0$ with regard to the values of $\phi_{\mathrm{ex}}$ in Fig. 1e.

While precise determination of populations is beyond reach in the case of fast exchange, semi-quantitative estimates are nevertheless possible. $\phi_{\mathrm{ex}}$ values in MPD-ub are consistent with $p_{\beta \mathrm{I}} \approx 5-10 \%$ and $\Delta \delta$ on the order of a fraction of ppm, as can be expected for the analyzed residues that are located at the periphery of the $\beta$-turn. This $p_{\beta I}$ estimate is supported by our MD simulations (see below). It is further reasonable to assume that $\Delta \delta$ values are approximately the same for the MPD-ub and cubic-PEG-ub experiencing the same exchange process (interconversion between $\beta I$ and $\beta$ II). If we make this assumption, then based on $\phi_{\text {ex }}$ results we can estimate that the populations of minor species in the cubic-PEG-ub crystal is $p_{\beta I I} \approx 20-50 \%$. Note that the population of minor species cannot exceed $50 \%$, which provides in this context a useful boundary. Supplementary Table 1 provides some example calculations relating the minorstate populations in the two crystals.

MD simulations using explicit crystal lattices have previously been shown to successfully reproduce experimental ssNMR and XRD data, and they may contribute valuable information about mechanistic details of motion that are difficult to access experimentally $6,35,48-50$. We simulated an explicit arrangement of ubiquitin molecules according to the crystal lattices of MPD-ub and cubic-PEG-ub, built of 24 and 48 ubiquitin molecules, respectively, in the presence of interstitial water over a time interval of $2 \mu \mathrm{s}$. The explicit simulation of a large number of ubiquitin molecules improves the sampling statistics considerably. In addition, we performed a $10 \mu$ s simulation of ubiquitin in solution, which we find to be in excellent agreement with a recent $1 \mathrm{~ms}$ trajectory ${ }^{32}$. In all these trajectories, we observed multiple $\beta \mathrm{I} \leftrightarrow \beta \mathrm{II}$ transitions, enabling us to extract the relative populations of the two conformations, which are reported in Table 1. The minor-state populations are qualitatively in good agreement with the estimates derived from BMCRD data, i.e., simulated MPD-ub has significantly lower minor-state population than cubic-PEG-ub (chain B), mirroring the lower $\phi_{\text {ex }}$ values. It should be noted that in the ssNMR spectra of cubic-PEG-ub the signals from the two inequivalent chains, A and B, overlap in most cases, making it impossible to collect two separate data sets. The experimental data, therefore, reflect an effective average of the dynamics in the two chains. With this caveat the $p_{\beta I I}$ populations found in the MD trajectory of cubic-PEG-ub are consistent with the experimental observations. 
Table 1 Populations and time scales of conformational exchange dynamics in the region encompassing residues L50-L56 and the adjacent $\mathbf{N}$-terminal part of the $\alpha$-helix

\begin{tabular}{|c|c|c|c|c|}
\hline & \multicolumn{2}{|l|}{ MD } & \multicolumn{2}{|c|}{ NMR (Bloch-McConnell relaxation dispersion data) } \\
\hline & BII population (\%) & Time constant $\tau_{\mathrm{ex}}(\mu \mathrm{s})$ & BII population (\%) & Time constant $\tau_{\mathrm{ex}}(\mu \mathrm{s})$ \\
\hline Solution & 3 & 2.5 & $3^{a}$ & $6^{a}$ \\
\hline Chain $\mathrm{A}$ & 9 & 1.1 & $\sim 20-50^{b}$ & $125 \pm 45$ \\
\hline Chain B & 43 & 0.4 & & \\
\hline
\end{tabular}

The time scale of the exchange process in the MD simulations tends to be faster than observed experimentally (Table 1), suggesting that the force field employed in this study does not accurately reflect the transition-state free-energy barrier. Similar findings have been reported before ${ }^{51}$. This observation is perhaps not surprising, given the limited accuracy of MD models (note that force fields have been parameterized primarily against experimental equilibrium data, and not against kinetic data). From the perspective of this study the elevated exchange rates are to some degree advantageous since this improves the statistical sampling of the $\beta \mathrm{I} / \beta \mathrm{II}$ equilibrium.

Thus, NMR and MD techniques independently indicate that the relative populations of $\beta \mathrm{I}$ and $\beta \mathrm{II}$ conformers differ considerably in different crystals and in solution. The $\beta \mathrm{I}: \beta \mathrm{II}$ populations span the wide range from 10:90 (MPD-ub) to 57:43/ 91:9 (cubic-PEG-ub) and 97:3 (solution).

Molecules in cubic-PEG-ub undergo tens-of-microseconds rocking motion. We also recorded NERRD experiments on cubic-PEG-ub crystals, in order to obtain additional insight into $\mu$ s dynamics in this crystal form. It shall be reminded that in MPD-ub only few residues (in particular D52 and R54; see Fig. 2a, b) show increased $R_{1 \rho}$ close to the rotary resonance condition (i.e., non-flat NERRD profiles), and that the time scale obtained from a fit of these data is in excellent agreement with Bloch-McConnell RD data. In contrast to this finding, essentially all residues in cubic-PEG-ub exhibit non-flat NERRD profiles, as shown in Fig. 3 and Supplementary Fig. 6, and the $R_{1 \rho}$ values are generally higher than those observed in MPD-ub. Given that this trend is observed for all residues, we ascribe the underlying motion to a global process involving the entire molecule, such as an overall "rocking" motion of the ubiquitin molecules in the crystal. Overall rocking motion is visible not only in $R_{1 \rho}$ measurements, but is also expected to contribute to line widths in ssNMR experiments. This expectation is indeed confirmed by ${ }^{1} \mathrm{H}$ and ${ }^{15} \mathrm{~N}$ coherences life times, which differ markedly between the two crystal forms studied here (Supplementary Fig. 7). We have proposed the existence of such overall motions in cubic-PEG-ub recently, based on the observation of elevated $R_{1 \rho}$ rate constants in cubic-PEG-ub compared to MPD-ub ${ }^{35}$, although the time scale could be estimated only very roughly (ca. $100 \mathrm{~ns}$ to $100 \mu \mathrm{s}$ ).

In order to obtain quantitative insight into rocking motion, we have performed a common fit of a dynamic model with a global time scale to NERRD data from 22 well-resolved residues in cubic-PEG-ub, excluding residues in the $\beta$-turn region, since for those residues one expects superposition of local and global motion. The best-fit time scale is of the order of tens of microseconds (Fig. 3d). Residue-wise motional amplitudes, expressed in terms of a two-site jump model, are in the range $3-5^{\circ}$ (Supplementary Fig. 6). Such a relatively small amplitude is expected for an overall-rocking motion within the confines of the lattice; MD simulations revealed an amplitude of overall motion in cubic-PEG-ub of $\sim 6-8^{\circ}$, although this amplitude is likely exaggerated due to simultaneous small drift (melting) of the crystal lattice ${ }^{35}$.

It is interesting to note that the tens-of-microseconds time scale of overall motion, found experimentally, is similar to the time scale of the $\beta$-turn conformational exchange in cubic-PEGub $(125 \pm 45 \mu \mathrm{s})$. This similarity may be fortuitous, but it might also point to some correlation between the two processes, i.e., the local and the overall motion. Such possibility is discussed in what follows.

Intermolecular contacts that alter conformational equilibria. As noted above, the $\beta \mathrm{I}$ conformation in ubiquitin represents a "default" state, which is found in solution and in the majority of crystal structures. At the same time the $\beta I I$ conformation has also been observed in a number of crystals, such as MPD-ub ${ }^{46}$ and complexes of ubiquitin (see references in Sidhu et al. $^{52}$ ). This leads us to conclude that the crystalline environment can act as a switch converting the protein from $\beta$ I to $\beta$ II. Our MD simulations of ubiquitin crystals are well suited to identify intermolecular interactions that are responsible for this transformation.

In our initial analysis of the MPD-ub trajectory we have identified two such putative intermolecular contacts. One is the four-way junction involving a pair of D52 side chains and a pair of K63 side chains, all of which belong to different ubiquitin molecules but come together in the crystal lattice (Fig. 4a). The other is the intermolecular hydrogen bond between the (neutral) carboxyl groups of E64 and E24, which appears to stabilize the coupling between E24 and G53 (Fig. 4b). According to the MD simulation data, both of these interactions show clear preference for the $\beta \mathrm{II}$ over the $\beta \mathrm{I}$ form.

However, additional simulations convinced us that this is the case of correlation rather than causation. Specifically, we have recorded two separate 2- $\mu$ s-long trajectories of MPD-ub crystals, one of which carried K63A mutation and the other E64A mutation. As it turned out, these trajectories produced exactly the same proportion of $\beta \mathrm{I}$ to $\beta \mathrm{II}$ species as the wild-type crystal simulation. This led us to conclude that the intermolecular contacts involving K63 and E64 side chains are not the key to the $\beta$-turn conformation.

At the same time, we have found that an E24A mutation produced a complete reversal of $\beta$ II to $\beta$ I ratio compared to the wild-type simulation, from $90 \%: 10 \%$ to $9 \%: 91 \%$. In other words, E24A mutation has restored the "default" state of the system, which is dominated by $\beta \mathrm{I}$. Apparently it is the hydrogen bond between the carboxyl group of E24 and the backbone amide group in G53 which is directly responsible for prevalence of the $\beta I I$ form in the wild-type MPD-ub crystal. But this hydrogen 


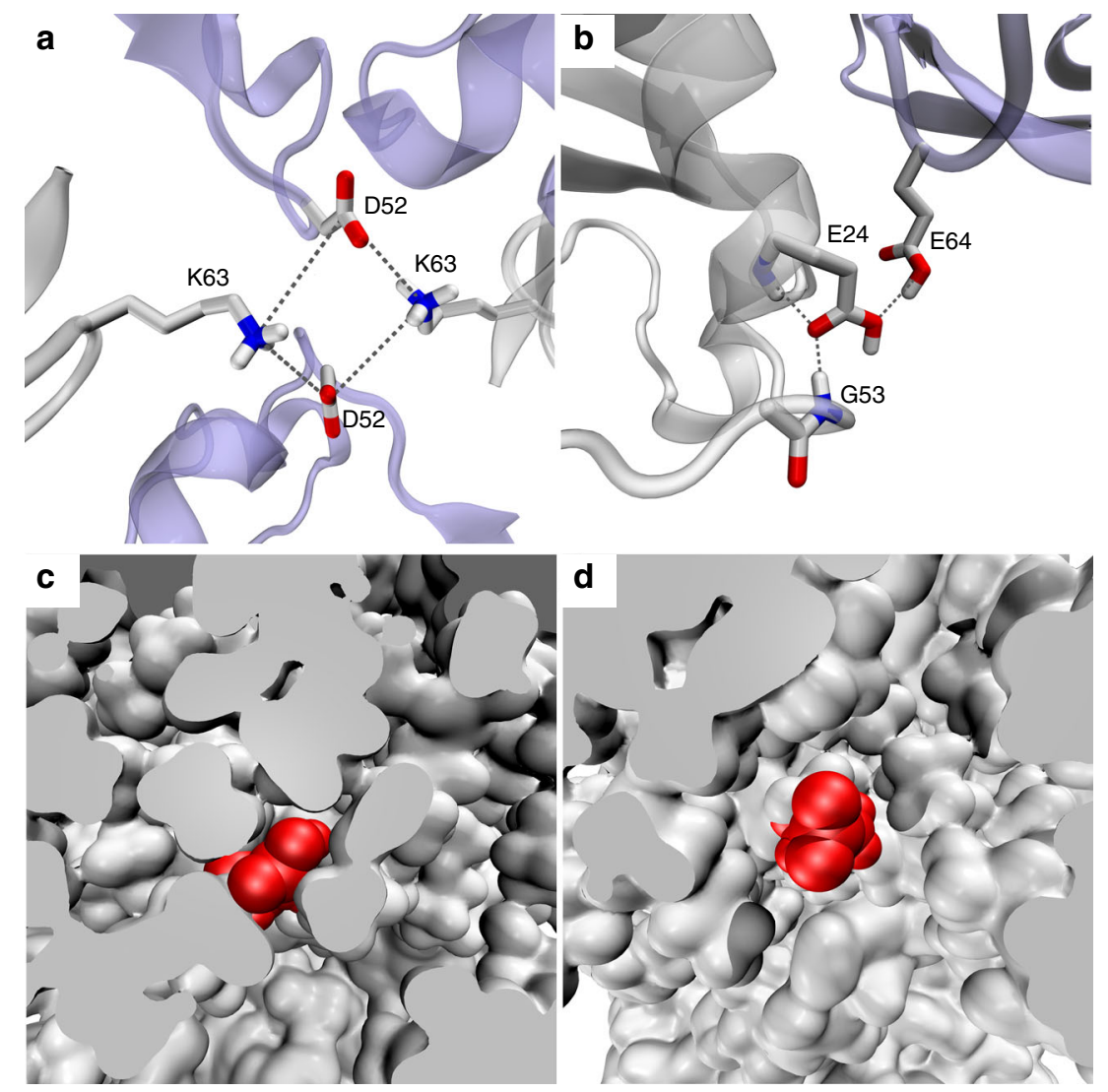

Fig. 4 Intermolecular and intramolecular interactions that affect $\beta$-turn motion. $\mathbf{a}$, $\mathbf{b}$ Intermolecular interactions involving the $\beta$-turn region in MPD-ub. Neighboring molecules are shown in light blue. Simulations of ubiquitin mutants suggest that these interactions are not responsible for causing the preference for $\beta$ II, see text. c, d The surrounding of E24 side chain in different crystal forms of ubiquitin. c A rare frame from the MPD-ub trajectory where E24 side chain (painted red) is projected toward solvent. d A typical frame from cubic-PEG-ub trajectory where E24 from ubiquitin molecule chain A is immersed into solvent

bond is an intramolecular hydrogen bond-so how is it related to crystal packing?

Inspection of the trajectories of ubiquitin in solution and in the cubic-PEG-ub crystal revealed that in these simulations the side chain of E24 is mostly immersed in solvent. In solution, E24 side chain is solvated $65 \%$ of the entire simulation time. In cubicPEG-ub, it is solvated $62 \%$ of the time for chain A and $52 \%$ of the time for chain B. In contrast, it is solvated at the level of only $11 \%$ in the MPD-ub simulation (Table 2).

As it turns out, the packing of ubiquitin molecules in the MPD$\mathrm{ub}$ crystal lattice is such that E24 side chain does not have the space to extend outwards. This is illustrated in Fig. 4c, d, which compare the volume of space available to E24 side chain in MPD-ub and in cubic-PEG-ub crystals. In particular, the intrinsically favorable rotameric state $\left(\chi_{1}=180^{\circ}, \chi_{2}=180^{\circ}\right)$ that is prominent in other simulations, is completely obstructed in MPD-ub. We conclude that typically the E24 side chain enjoys the entropic benefit of being immersed in solvent, but in MPD-ub it is denied this opportunity and therefore opts for the hydrogen bond with G53.

The propensity of the carboxylic group in E24 to form a hydrogen bond with the G53 amide group is also influenced by its protonation state. The MPD-ub crystal has been obtained from a mother liquor at $\mathrm{pH}$ 4.2. Under these conditions the carboxylic group of E24, as positioned in the crystallographic structure, is expected to be mostly protonated ${ }^{53}$. To elucidate the role of this factor, we have altered E24's status from neutral to anionic and then recorded an additional 2- $\mu$ s-long simulation of the MPD-ub crystal. As it turns out, in this trajectory E24 is partially recruited into both intramolecular and intermolecular salt bridges (Table 2).
This happens at the expense of the E24-G53 hydrogen bond, which drops to the level of $28 \%$. Accordingly, the ratio of $\beta \mathrm{II}$ to $\beta \mathrm{I}$ in this trajectory changes from $90 \%: 10 \%$ to $71 \%: 27 \%$. This finding supports the hypothesis that the protonation state of E24 also plays a significant role in the stabilization of the $\beta$ II species in MPD-ub crystal.

Potential coupling between rocking and $\beta I / \beta I I$ exchange. As discussed above, cubic-PEG-ub crystal is characterized by significant amount of rocking and, at the same time, extensive conformational exchange in the $\beta \mathrm{I} / \beta \mathrm{II}$ region. Furthermore, the characteristic time constants of these two motional processes turn out to be similar. Is this a mere coincidence, or does it point toward a certain common underlying mechanism?

Inspection of the cubic-PEG-ub trajectory led us to identify one potential coupling mechanism. This mechanism involves an ion pair between K11 (chain A) and D52 (chain B). In the crystal structure these two side chains are positioned far apart (Fig. 5a). However, during the MD simulation they frequently form a salt bridge (Fig. 5b). The formation of this salt bridge is associated with (i) slight reorientation of protein molecules in the crystal lattice and (ii) $\beta \mathrm{I} \rightarrow \beta \mathrm{II}$ transition mediated by residue D52 in the $\beta$-turn. Thus the reorientational dynamics of the protein (rocking motion) may be correlated with conformational exchange. This hypothetical scenario is illustrated in the Supplementary Movie 1.

We have yet been able to obtain only partial confirmation for this scenario. The MD data indicate that $\mathrm{K} 11_{\text {chainA }}-\mathrm{D} 52_{\text {chainB }}$ salt bridge in the cubic-PEG-ub crystal favors $\beta$ II conformation over 
Table 2 Important interactions involving E24 side-chain carboxylic group as characterized by their presence in the MD simulations

\begin{tabular}{llllll} 
E24 side chain contacts & Solution (\%) & MPD-ub & & \multicolumn{2}{c}{ Cubic-PEG-ub } \\
\cline { 3 - 4 } & & E24 neutral (\%) & E24 charged (\%) & Chain A (\%) & Chain B (\%) \\
\hline E24-water & 65 & 11 & 4 & 62 & 52 \\
E24- $\mathrm{K} 27\left(\mathrm{NH}_{3}{ }^{+}\right)$ & 34 & 2 & 20 & 26 & 27 \\
E24-G53(NH) & 0.5 & 71 & 28 & 1 & 2 \\
E24- $\mathrm{K} 63\left(\mathrm{NH}_{3}{ }^{+}\right)$ & 0 & 1 & 64 & 0 & 0
\end{tabular}

The contacts involving K27 and G53 are intramolecular, the contact involving K63 is intermolecular. The numbers in each column do not always add up to $100 \%$ because E24 also forms certain other contacts (both intermolecular and intramolecular) or, otherwise, some of the listed interactions can occur simultaneously

$\beta$ I (by a factor of 4.1 considering the trajectory at hand). In order to probe this effect experimentally, we have prepared cubic-PEG$\mathrm{ub}$ crystals of K11A ubiquitin, and performed BMCRD experiments. We saw a small but detectable decrease in RD amplitudes for residues in the $\beta$-turn region in the K11A ubiquitin crystal compared to WT crystal (Supplementary Fig. 10). This reduced $\mathrm{RD}$ effect may indeed point to a decreased population of minor $\beta$ II state in K11A.

On the other hand, our attempts to detect the link between $\mathrm{K} 11_{\text {chain }}-\mathrm{D} 52_{\text {chainB }}$ and rocking motion proved to be unsuccessful. The MD simulation of K11A crystal failed to produce any significant evidence that on-off $\mathrm{K} 11_{\text {chainA }}-\mathrm{D} 52_{\text {chainB }}$ salt bridge drives rocking dynamics. In part, this negative result can be attributed to poor convergence of the MD trajectories, which are affected by gradual "melting" of the crystal lattice (discussed by us previously $^{35}$; see Supplementary Fig. 12). One should also bear in mind that ubiquitin molecules in the crystals are held together by a complex network of intermolecular interactions involving several dozen hydrogen bonds and salt bridges (Supplementary Figs. 8 and 9). In this situation the effect from the single-point mutation, K11A, may be masked; moreover, the consequences of the mutation may be more complex and differ from what has been expected. The experimental NERRD data from the sample of K11A ubiquitin also could not detect the expected decrease in rocking within the experimental precision (Supplementary Fig. 11).

Although in this specific case we could not confirm that conformational exchange is coupled to rocking, this hypothesis deserves further investigation. It is envisioned that local conformational rearrangements may lead to "repacking" of protein molecules in the crystal lattice, entailing small overall translation and reorientation (i.e., causing rocking motion).
Conversely, rocking motion may lead to subtle changes in the pattern of crystal contacts, triggering local conformational transitions. In general, it should not be surprising that a molecule that is conformationally labile produces a poor crystal which suffers, inter alia, from rocking dynamics.

Probing relaxation dispersion effects. MD simulations on a microsecond time scale can be used to model BMCRD effects. For this purpose MD coordinates are fed, frame by frame, into chemical shift prediction programs such as SHIFTX ${ }^{54}$. In a situation when the calculated chemical shift $\delta\left({ }^{15} \mathrm{~N}\right)$ shows a pattern of modulation on the $\mu$ s time scale, one may expect to experimentally detect $\mathrm{RD}$ effects at the respective site ${ }^{55}$. Ultimately, this approach makes it possible to uncover the dynamic mechanism behind the observable RD profiles.

We have used this method to probe the backbone ${ }^{15} \mathrm{~N}$ sites in MPD-ub and cubic-PEG-ub trajectories. In MPD-ub, relaxation dispersion effects have been predicted for sites in the $\beta$-turn experiencing $\beta \mathrm{I} \leftrightarrow \beta \mathrm{II}$ exchange, as well as sites at the $\mathrm{N}$-terminal end of the first $\alpha$ helix that are hydrogen-bonded to this $\beta$-turn. One example of such behavior, residue R54 from MPD-ub, is illustrated in the Supplementary Movie 2, demonstrating that the $\beta \mathrm{I} \leftrightarrow \beta \mathrm{II}$ transition is accompanied by a ${ }^{15} \mathrm{~N}$ chemical-shift change in R54.

Similarly, the analysis of the cubic-PEG-ub trajectory suggests that Bloch-McConnell dispersions can occur in the $\beta$-turn and its coupled sites (particularly, in the chain B molecules). In addition, we have also found several sites where the origin of predicted RD effects is different. One example of such distinctive behavior is residue H68. Focusing on one specific ubiquitin molecule in cubic-PEG-ub trajectory, we observe H68 side chain engaged in

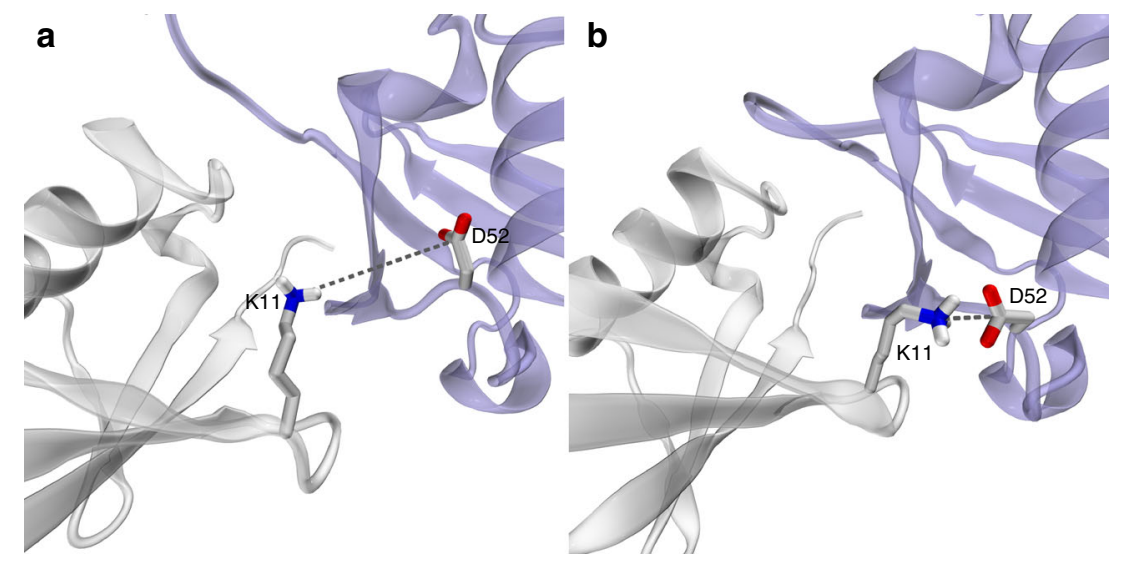

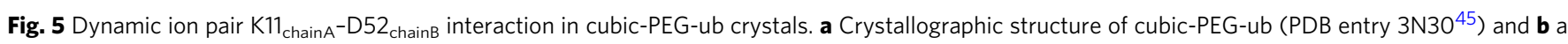
selected frame from cubic-PEG-ub trajectory. The dynamic disorder in residue $\mathrm{K} 11_{\text {chain }}$ is reflected in its crystallographic temperature factors; this residue's side chain has not been modeled in our recent structure $4 \mathrm{XOL}$ 
a

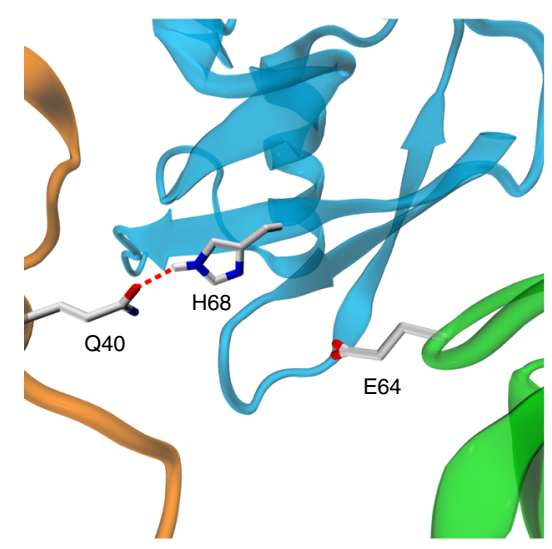

b

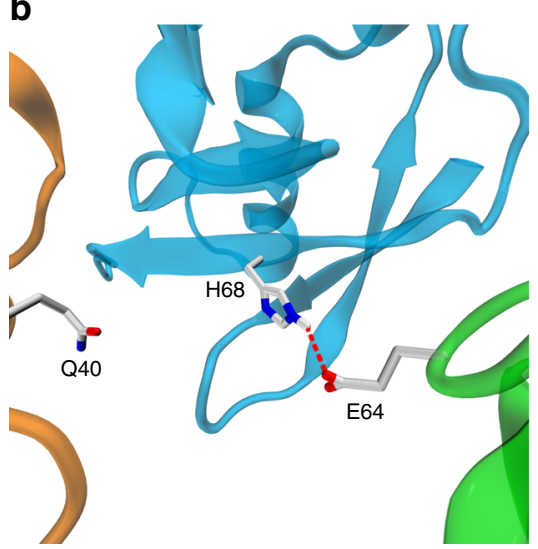

c

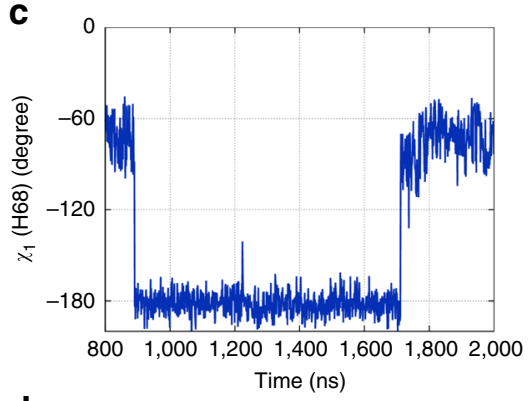

d

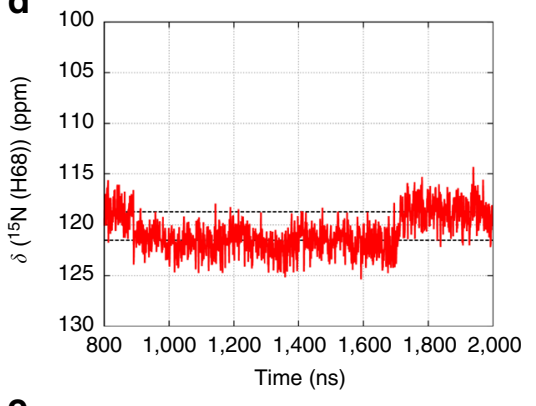

e

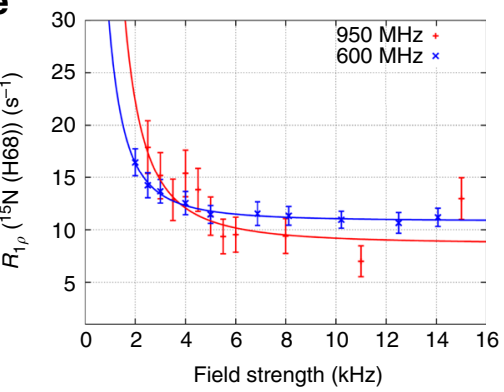

Fig. 6 Microsecond time scale dynamics of residue $\mathrm{H} 68$ (chain A) in cubic-PEG-ub crystals. a, $\mathbf{b}$ Intermolecular hydrogen bonds alternatively formed between $\mathrm{H} 68$ and Q40 or E64 as seen in the MD simulation of cubic-PEG-ub. c, d Time variation of side-chain torsional angle $\chi_{1}(t)$ and main-chain ${ }^{15} \mathrm{~N}$ chemical shift $\delta(t)$ for residue $\mathrm{H} 68$ in the selected ubiquitin molecule from the simulated unit crystal cell. e Experimental relaxation dispersion data for residue $\mathrm{H} 68$ (chain unassigned) in the cubic-PEG-ub crystal; also shown are the results of fitting using fast exchange model (continuous lines). Error bars of $R_{1 \rho}$ values were determined from Monte Carlo analysis. The motional time scale according to the experimental data, $56 \mu \mathrm{s}$, is one-to-two orders of magnitude slower than suggested by the MD simulations (the same is true for other manifestations of $\mu$ s dynamics in ubiquitin crystals). The animated version of this graph is available as Supplementary Movie 3

hydrogen bond with Q40 side chain from a neighboring molecule (Fig. 6a). Later during the simulation H68 side chain swings sideways and forms hydrogen bond with side chain E64 that belongs to another neighboring molecule (Fig. 6b). After ca. $0.8 \mu \mathrm{s}$ it makes a reverse transition, reestablishing hydrogen bond with Q40, and remains in this conformation until the end of the trajectory.

The rotameric transitions of $\mathrm{H} 68$ (from $\chi_{1}=-60^{\circ}$ to $180^{\circ}$ and back) cause modulation of the backbone chemical shifts in this residue. For the ${ }^{15} \mathrm{~N}$ spin the amplitude of modulation is calculated to be $2.1 \mathrm{ppm}$ (corresponding to the gap between the two dashed horizontal lines in Fig. 6d). This is a sizable effect, which can be reliably reproduced by chemical shift prediction software. Therefore the computational analysis points toward possible RD effect at the amide site in residue H68. This prediction is borne out by the experimental data illustrated in Fig. 6 e.

Of interest, there is a theoretical possibility that conformational exchange in H68 is influenced by rocking motion (cf. previous section). Indeed, slight reorientation of the central protein molecule relative to its neighbors should favor either H68-Q40 or H68-E64 interaction depending on the direction of reorientational fluctuation (Fig. 6a, b). In this connection, it is noteworthy that RD effects have been experimentally detected at this site in the cubic-PEG-ub crystal form that is affected by rocking, but not in the MPD-ub crystal that is immune to rocking (Supplementary Fig. 3).

\section{Discussion}

The advent of novel experimental and theoretical tools to decipher the heterogeneity of structures co-existing in crystals has sparked increased interest in molecular motions of crystalline proteins and the role of crystal packing in the context of dynamics. Here we have obtained a comprehensive portrayal of us dynamics in different crystal forms of ubiquitin. Experimental ${ }^{15} \mathrm{~N} R_{1 \rho}$ relaxation data, specifically the combination of BMCRD and NERRD analyses, produced evidence of two major motional modes: conformational exchange in the $\beta$-turn formed by residues E51-R54 and overall rocking. The characteristic time scale of a local $\beta$-turn interconversion motion in the two investigated crystals is ca. $100 \mu$ s, more than one order of magnitude slower than in solution. Overall rocking motion entails small reorientational fluctuations of the protein molecules within the confines of the crystal lattice and affects molecules in one of the two crystal forms, cubic-PEG-ub, much more significantly than in the other, MPD-ub. The amplitude of the rocking motion in cubic- 
PEG-ub was determined to be ca. $4^{\circ}$, and the time constant was found to be on the scale of tens of microseconds. These results, as well as other findings reported in our study, show that $\mu$ s dynamics is dependent on the environment.

MD simulations, including mutant simulations, can rationalize these effects. In particular, we can tentatively explain why MPDub prefers the type-II $\beta$-turn conformation. The acquired experimental evidence together with MD data hint at the possibility that internal conformational dynamics may be coupled to the rocking motion. Elucidating this connection in protein crystals is a daunting task. In particular, accurately modeling something as subtle and fickle as crystal contacts is a major challenge for the existing MD methodology. Nevertheless, we expect that future advances in the field of MD simulations should eventually make it possible. The identification of intermolecular interactions that control overall motion, either directly or through their involvement in internal conformational exchange, might open future possibilities for rational design of mutant proteins that could reduce the effect of rocking and thus improve diffraction quality.

It is known that internal dynamics undermine the quality of $\mathrm{X}$ ray diffraction data. Crystallographers go a great length in order to reduce the level of dynamics in their samples (e.g., by engineering point mutations or by introducing ligands). It has not been fully appreciated, however, that internal conformational exchange may be connected to rocking motion, which can be even more detrimental for crystallographic analyses. We have shown here that both internal and overall motions can be probed by ssNMR spectroscopy and MD simulations. It is envisaged that both methods will be successfully exploited in future to either help in designing of stable crystals or, conversely, to assist in Xray diffraction-based studies of protein motions.

\section{Methods}

Sample preparation. Uniformly ${ }^{2} \mathrm{H},{ }^{15} \mathrm{~N}$-labeled human ubiquitin (without any affinity tag) was overexpressed in E. coli, and purified using anion exchange and size exclusion chromatographies. In order to have a dilute ${ }^{1} \mathrm{H}$ spin network and thus minimize the possible perturbing effect of remote ${ }^{1} \mathrm{H}$ spins on the relaxation of amide ${ }^{15} \mathrm{~N}$, the protein was dissolved in $\mathrm{H}_{2} \mathrm{O}: \mathrm{D}_{2} \mathrm{O}(20: 80)$ mixture at $\mathrm{pH} 7$, resulting in isotope incorporation of ${ }^{1} \mathrm{H} /{ }^{2} \mathrm{H}$ at exchangeable sites at a ca. 20:80 ratio, and then lyophilized. Solutions used for crystallization were prepared by dissolving this lyophilized protein in buffers containing the same $\mathrm{H}_{2} \mathrm{O}: \mathrm{D}_{2} \mathrm{O}$ ratio. All specified $\mathrm{pH}$ values below are corrected for the glass electrode isotope effect ${ }^{56}$. For generating MPD-ub crystals, lyophilized ubiquitin was dissolved at $20 \mathrm{mg} \mathrm{ml}^{-1}$ concentration in buffer A ( $20 \mathrm{mM}$ ammonium acetate, $\mathrm{pH} 4.2$ ). Buffer B (50 mM citrate, $\mathrm{pH} 4.2$ ) was mixed with MPD at a volume ratio of 40:60. A volume of $500 \mu \mathrm{l}$ of this solution was deposited in the wells of sitting-drop crystallization plates. The protein solution $(37 \mu \mathrm{l})$ was mixed with the buffer B/MPD solution $(10 \mu \mathrm{l})$ and deposited as sitting-drop in the crystallization plate $(24$-well plates with $1.5 \mathrm{ml}$ reservoir volume). The plate was covered with sealing tape and kept at $4{ }^{\circ} \mathrm{C}$; crystals appeared after 1-2 weeks, and were filled into a $1.6 \mathrm{~mm}$ Agilent or $1.3 \mathrm{~mm}$ Bruker rotor using an ultracentrifuge device; caps were glued with epoxy glue to avoid dehydration. Cubic-PEG-ub crystals were prepared with the same equipment, but the protein was dissolved in buffer $\mathrm{C}(20 \mathrm{mM}$ ammonium acetate $\mathrm{pH} 4.2)$ and mixed with buffer D (200 mM zinc acetate, $100 \mathrm{mM} \mathrm{MES,} \mathrm{pH} \mathrm{6.3,} \mathrm{20 \%} \mathrm{w/v} \mathrm{PEG}$ $3350)$ and deposited as sitting-drop. The mother liquor reservoir was filled with $500 \mu$ l of buffer D. Crystals were obtained at $20^{\circ} \mathrm{C}$ after circa 3-4 weeks.

Solid-state NMR and data analysis. ${ }^{15} \mathrm{~N} R_{1 \rho}$ relaxation measurements were performed on (i) a $600 \mathrm{MHz}(14.1 \mathrm{~T})$ Agilent VNMRS spectrometer (only BMCRD experiment) equipped with a $1.6 \mathrm{~mm}$ triple-resonance MAS probe tuned to ${ }^{1} \mathrm{H},{ }^{13} \mathrm{C},{ }^{15} \mathrm{~N}$ frequencies and (ii) a $600 \mathrm{MHz}$ and (iii) a $950 \mathrm{MHz}(22.3 \mathrm{~T})$ Bruker Avance 2 spectrometer; the latter two were equipped with $1.3 \mathrm{~mm}$ MAS probes tuned to ${ }^{1} \mathrm{H},{ }^{13} \mathrm{C},{ }^{15} \mathrm{~N}$ frequencies with an auxiliary ${ }^{2} \mathrm{H}$ coil. Resonance assignments of MPD-ub and cubic-PEG-ub have been reported elsewhere ${ }^{35}$. BMCRD experiments at $600 \mathrm{MHz}$ were performed at $39.5 \mathrm{kHz}$ MAS, while BMCRD data at 950 $\mathrm{MHz}$ were collected at $50 \mathrm{kHz}$ MAS. BMCRD data of MPD-ub at $600 \mathrm{MHz}$ have been reported before ${ }^{34}$. NERRD data on both MPD-ub and cubic-PEG-ub were recorded on the $600 \mathrm{MHz}$ (Bruker) spectrometer at a MAS frequency of 44.053 $\mathrm{kHz}$. In order to enhance resolution, the BMCRD data on cubic-PEG-ub at 950 $\mathrm{MHz}$ were recorded as a series of proton-detected $3 \mathrm{D}$ hCONH-based experiments using a two-point approach with spin-lock periods of 1 and $50 \mathrm{~ms}$. The 3D approach enabled us to resolve the otherwise overlapped I23 resonance. All other data were collected with $2 \mathrm{D}$ proton-detected hNH correlation experiments, shown in ref. ${ }^{57}$, using a series of relaxation delays (typically 8 points up to $125 \mathrm{~ms}$ ). In all experiments, cross-polarization steps were used for transfer, with $\sim 85 \mathrm{kHz}{ }^{1} \mathrm{H}$ RF field, and a ${ }^{15} \mathrm{~N}$ RF field adjusted to the $n=1$ Hartmann-Hahn condition. Peak volumes in the individual 2D (3D) spectra were measured in NMRView (OneMoon Scientific), and relaxation rate constants were derived from monoexponential fits, using fitting routines written in python/numpy/scipy language. On-resonance $R_{1 \rho}$ relaxation rate constants were derived from the measured rate constants taking into account the resonance offset of the given cross-peak from the RF-field carrier $\Omega$ and the longitudinal relaxation-rate constant $R_{1}$ as described elsewhere ${ }^{34}$. This correction is fairly small, and visible primarily for low (less than $\sim 3 \mathrm{kHz}$ ) RF fields. Experiments at $950 \mathrm{MHz}$ were measured twice, with two different carrier settings (112 and $125 \mathrm{ppm}$ ), and the data reported for each residue correspond to the experiment with the carrier position closest to the resonance frequency. Error bars of relaxation rate constants were estimated from standard Monte Carlo simulations ${ }^{58}$ : the parameters obtained from fitting the experimental data set were used to generate a set of ca. 500 synthetic data sets, in which the peak intensities were randomly varied, relative to the back-calculated point, within three times the spectral noise level. These synthetic data sets were subsequently fitted in an identical way as the original data set, resulting in 500 fitted decay rate constants. The reported error bars correspond to the standard deviation of these rate constants.

Fits of a two-state exchange model as reported by Meiboom ${ }^{59}$, to the BMCRD data were obtained from the program relax, version $4.0^{60}$. For the fits shown in Fig. 1, we chose five residues for which data of both crystals were available: I23 (only $950 \mathrm{MHz}$, as no $600 \mathrm{MHz}$ data were available for cubic-PEG-ub) and V26, $\mathrm{K} 27, \mathrm{~T} 55, \mathrm{D} 58(600$ and $950 \mathrm{MHz})$. We have investigated how the choice of residues, and the choice of static magnetic field strengths influences the outcome of these fits; we find that using different subgroups of residues in the $\beta$-turn region, or including other residues in the fits where available (e.g., T22 in MPD-ub) does not significantly alter the results (Supplementary Fig. 5).

For the analysis of NERRD data, we used numerical simulations, rather than the analytical equations (Eqs. (1) and (2)). We prefer numerical simulations, because Eqs. (1) and (2) report accurately on the initial slope of relaxation decays, but fail to describe the multi-exponential behavior of the relaxation due to crystallite averaging. It has been proposed to determine the initial slope experimentally ${ }^{61}$, but we instead choose (i) to use monoexponential fits of the experimental data and (ii) to interpret the resulting rate constants by using numerical simulations of decays, imitating the experimental setup as closely as possible. A similar type of data analysis, involving numerical simulations of spin relaxation, has been used previously ${ }^{40}$. Details about the numerical simulations of the spin evolution and numerical fits are described in the Supporting Information.

MD simulations. The 2- $\mu$ s-long MD simulations of MPD-ub crystal (24 ubiquitin molecules) and cubic-PEG-ub crystal (48 ubiquitin molecules) recorded under Amber ff99SB*-ILDN force field ${ }^{22-64}$ in the presence of SPC/E water ${ }^{65}$ are the same as reported by us previously ${ }^{35}$, and described in detail in the Supplementary Methods. Analogous 2- $\mu$ s-long trajectories have been recorded for MPD-ub crystals containing single-residue mutations E24A, G53A, K63A, E64A, or a negatively charged form of E24. For cubic-PEG-ub form, we have recorded the duplicate 2- $\mu \mathrm{s}$ trajectory of the wild-type ubiquitin crystal and the additional trajectories for crystals with K11A, K11A (chain A only), and G53A mutations. All alanine substitutions did not create any steric conflicts in the initial crystal coordinates. The compound statistics from each crystal trajectory is equivalent to $2 \times 24=48$ or $2 \times$ $48=96 \mu$ s of ubiquitin dynamics. (Strictly speaking, many short trajectories are not equivalent to one long one, but in our case the simulation length is sufficient to sample the events of interest, i.e., conformational exchange and rocking). The trajectories exhibit a certain amount of drift, as has been discussed by us earlier; ${ }^{35}$ the drift is essentially a reflection of rocking motion that becomes progressively worse during the course of the simulations. In addition, we have also recorded a $1-\mu s-l o n g$ trajectory of cubic-PEG-ub crystal, where $\mathrm{C}^{\alpha}$ atoms within the secondary structure of ubiquitin have been restrained to their respective positions in the crystallographic structure by means of harmonic restraints with force constant $5 \mathrm{kcal} \mathrm{mol}^{-1} \AA^{-2}$. The rocking motion was largely suppressed in this trajectory. However, this trajectory featured a significant proportion of distinctive $\beta$ II' species, which appears to be an artifact. Therefore the restrained simulation was discontinued. As a control, we have also recorded 2- $\mu$ s-long trajectories of MPD-ub crystal and cubic-PEG-ub crystal (wild-type, K11A, K11A chain A only, and G53A) using newer ff14SB force field ${ }^{66}$. Finally, the previously reported solution trajectory of ubiquitin has been extended to $10 \mu \mathrm{s}$.

In processing the MD trajectories, we used geometric criteria to identify hydrogen bonds (nitrogen-oxygen distance $<3.2 \AA$, angle from $130^{\circ}$ to $180^{\circ}$ ) and salt bridges (at least one nitrogen-oxygen distance $<4 \AA$, centroid distance $5 \AA)^{67,68}$. Chemical shifts have been calculated by processing the MD coordinates using the program SHIFTX ${ }^{54}$. This program has essentially the same accuracy as SHIFTX + module from SHIFTX $2{ }^{69}$, while relying on a simpler parameterization that nicely captures the dependence of ${ }^{15} \mathrm{~N}$ chemical shift on several essential structural variables. Given the limited accuracy of chemical shift prediction software, only the more pronounced modulation effects with $\Delta \delta\left({ }^{15} \mathrm{~N}\right)$ on the order 
of $1 \mathrm{ppm}$ can be reliably identified. Special tests have been performed to evaluate intermolecular contributions into chemical shifts; we have found that these contributions can be safely neglected for the problem at hand.

Data availability. Solid-state NMR relaxation data and MD simulation frames can be obtained from the corresponding authors upon request.

Received: 19 December 2016 Accepted: 7 June 2017

Published online: 27 July 2017

\section{References}

1. Burnley, B. T., Afonine, P. V., Adams, P. D. \& Gros, P. Modelling dynamics in protein crystal structures by ensemble refinement. Elife 1, e00311 (2012).

2. de Bakker, P. I. W., Furnham, N., Blundell, T. L. \& DePristo, M. Conformer generation under restraints. Curr. Opin. Struct. Biol. 16, 160-165 (2006).

3. Lang, P. T., Holton, J. M., Fraser, J. S. \& Alber, T. Protein structural ensembles are revealed by redefining X-ray electron density noise. Proc. Natl Acad. Sci. USA 111, 237-242 (2014).

4. Fraser, J. S. et al. Accessing protein conformational ensembles using roomtemperature X-ray crystallography. Proc. Natl Acad. Sci. USA 108, 16247-16252 (2011).

5. Van Benschoten, A. H. et al. Measuring and modeling diffuse scattering in protein X-ray crystallography. Proc. Natl Acad. Sci. USA 113, 4069-4074 (2016).

6. Wall, M. E. et al. Conformational dynamics of a crystalline protein from microsecond-scale molecular dynamics simulations and diffuse X-ray scattering. Proc. Natl Acad. Sci. USA 111, 17887-17892 (2014).

7. Mittermaier, A. \& Kay, L. E. New tools provide new insights in NMR studies of protein dynamics. Science 312, 224-228 (2006).

8. Mao, B., Tejero, R., Baker, D. \& Montelione, G. T. Protein NMR structures refined with Rosetta have higher accuracy relative to corresponding X-ray crystal structures. J. Am. Chem. Soc. 136, 1893-1906 (2014).

9. Bax, A. Weak alignment offers new NMR opportunities to study protein structure and dynamics. Protein Sci. 12, 1-16 (2003).

10. Ferrari, D. et al. Catalysis and electron transfer in protein crystals: the binary and ternary complexes of methylamine dehydrogenase with electron acceptors. Biochim. Biophys. Acta 1647, 337-342 (2003).

11. Pearson, A. R. \& Wilmot, C. M. Catching catalysis in the act: using single crystal kinetics to trap methylamine dehydrogenase reaction intermediates. Biochim. Biophys. Acta 1647, 381-389 (2003).

12. Ronda, L., Bruno, S., Bettati, S. \& Mozzarelli, A. Protein crystal microspectrophotometry. Biochim. Biophys. Acta 1814, 734-741 (2011).

13. Merli, A. et al. Enzymatic and electron transfer activities in crystalline protein complexes. J. Biol. Chem. 271, 9177-9180 (1996).

14. Mozzarelli, A. \& Rossi, G. L. Protein function in the crystal. Annu. Rev. Biophys. Biomol. Struct. 25, 343-365 (1996).

15. Sahu, S., Bhuyan, A., Majumdar, A. \& Udgaonkar, J. Backbone dynamics of barstar: a ${ }^{15}$ N NMR relaxation study. Proteins 41, 460-474 (2000).

16. Kordel, J., Skelton, N., Akke, M., Palmer, A. G. \& Chazin, W. Backbone dynamics of calcium-loaded calbinding- $\mathrm{D}_{9 \mathrm{k}}$ studied by two-dimensional proton-detected ${ }^{15} \mathrm{~N}$ NMR spectroscopy. Biochemistry 31, 4856-4866 (1992).

17. Powers, R., Clore, G., Garrett, D. \& Gronenborn, A. Relationships between the precision of high-resolution protein NMR structures, solution order parameters and crystallographic B factors. J. Magn. Reson. B 101, 325-327 (1993).

18. Fenwick, R. B., van den Bedem, H., Fraser, J. S. \& Wright, P. E. Integrated description of protein dynamics from room-temperature X-ray crystallography and NMR. Proc. Natl Acad. Sci. USA 111, E445-E454 (2014).

19. Agarwal, V., Xue, Y., Reif, B. \& Skrynnikov, N. R. Protein side-chain dynamics as observed by solution- and solid-state NMR spectroscopy: a similarity revealed. J. Am. Chem. Soc. 130, 16611-16621 (2008).

20. Haller, J. D. \& Schanda, P. Amplitudes and time scales of picosecond-tomicrosecond motion in proteins studied by solid-state NMR: a critical evaluation of experimental approaches and application to crystalline ubiquitin. J. Biomol. NMR 57, 263-280 (2013).

21. Yang, J., Tasayco, M. L. \& Polenova, T. Dynamics of reassembled thioredoxin studied by magic angle spinning NMR: snapshots from different time scales. J. Am. Chem. Soc. 131, 13690-13702 (2009).

22. Halle, B. Flexibility and packing in proteins. Proc. Natl Acad. Sci. USA 99, 1274-1279 (2002).

23. Eastman, P., Pellegrini, M. \& Doniach, S. Protein flexibility in solution and in crystals. J. Chem. Phys. 110, 10141-10152 (1999).

24. Stocker, U., Spiegel, K. \& van Gunsteren, W. On the similarity of properties in solution or in the crystalline state: a molecular dynamics study of hen lysozyme. J. Biomol. NMR 18, 1-12 (2000).

25. Rueda, M. et al. A consensus view of protein dynamics. Proc. Natl Acad. Sci. USA 104, 796-801 (2007).
26. Henzler-Wildman, K. \& Kern, D. Dynamic personalities of proteins. Nature 450, 964-972 (2007).

27. Massi, F. Microsecond timescale backbone conformational dynamics in ubiquitin studied with NMR $\mathrm{R}_{1 \rho}$ relaxation experiments. Protein Sci. 14, 735-742 (2005).

28. Hansen, D. F., Feng, H., Zhou, Z., Bai, Y. \& Kay, L. E. Selective characterization of microsecond motions in proteins by NMR relaxation. J. Am. Chem. Soc. 131 16257-16265 (2009).

29. Salvi, N., Ulzega, S., Ferrage, F. \& Bodenhausen, G. Time scales of slow motions in ubiquitin explored by heteronuclear double resonance. J. Am. Chem. Soc. 134, 2481-2484 (2012).

30. Smith, C. A. et al. Allosteric switch regulates protein-protein binding through collective motion. Proc. Natl Acad. Sci. USA 113, 3269-3274 (2016).

31. Mills, J. L. \& Szyperski, T. Protein dynamics in supercooled water: the search for slow motional modes. J. Biomol. NMR 23, 63-67 (2002).

32. Lindorff-Larsen, K., Maragakis, P., Piana, S. \& Shaw, D. E. Picosecond to millisecond structural dynamics in human ubiquitin. J. Phys. Chem. B 120, 8313-8320 (2016).

33. Tollinger, M., Sivertsen, A. C., Meier, B. H., Ernst, M. \& Schanda, P. Siteresolved measurement of microsecond-to-millisecond conformationalexchange processes in proteins by solid-state NMR spectroscopy. J. Am. Chem. Soc. 134, 14800-14807 (2012).

34. Ma, P. et al. Probing transient conformational states of proteins by solid-state $\mathrm{R}_{1 \rho}$ relaxation-dispersion NMR spectroscopy. Angew. Chem. Int. Ed. 53, 4312-4317 (2014).

35. Ma, P. et al. Observing the overall rocking motion of a protein in a crystal. Nat. Commun. 6, 8361 (2015).

36. Quinn, C. M. \& McDermott, A. E. Monitoring conformational dynamics with solid-state $\mathrm{R}_{1 \rho}$ experiments. J. Biomol. NMR 45, 5-8 (2009).

37. Quinn, C. M. \& McDermott, A. E. Quantifying conformational dynamics using solid-state $\mathrm{R}_{1 \rho}$ experiments. J. Magn. Reson. 222, 1-7 (2012).

38. Palmer, A. G. \& Massi, F. Characterization of the dynamics of biomacromolecules using rotating-frame spin relaxation NMR spectroscopy. Chem. Rev. 106, 1700-1719 (2006).

39. Kurbanov, R., Zinkevich, T. \& Krushelnitsky, A. The nuclear magnetic resonance relaxation data analysis in solids: general $R_{1} / R_{1 \rho}$ equations and the model-free approach. J. Chem. Phys. 135, 184104 (2011).

40. Smith, A. A., Testori, E., Cadalbert, R., Meier, B. H. \& Ernst, M. Characterization of fibril dynamics on three timescales by solid-state NMR J. Biomol. NMR 65, 171-191 (2016).

41. Lakomek, N.-A. et al. Microsecond Dynamics in Ubiquitin Probed by SolidState 15N NMR Spectroscopy R1 $\rho$ Relaxation Experiments under Fast MAS (60-110 kHz). Chem. Eur. J. doi:10.1002/chem.201701738 (2011).

42. Zinkevich, T., Chevelkov, V., Reif, B., Saalwächter, K. \& Krushelnitsky, A. Internal protein dynamics on ps to $\mu$ s timescales as studied by multifrequency 15N solid-state NMR relaxation. J. Biomol. NMR 57, 219-235 (2013).

43. Lamley, J. M., C. Öster, C., Stevens, R. A. \& Lewandowski, J. R. Intermolecular Interactions and Protein Dynamics by Solid-State NMR Spectroscopy. Angew. Chem. Int. Ed. 54, 15374-15378 (2015).

44. Ma, P. et al. Probing transient conformational states of proteins by solid-state

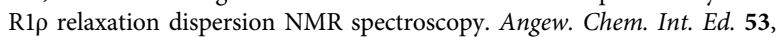
4312-4317 (2014).

45. Arnesano, F. et al. Crystallographic analysis of metal-ion binding to human Ubiquitin. Chem Eur. J. 17, 1569-1578 (2011).

46. Huang, K.-Y., Amodeo, G. A., Tong, L. \& McDermott, A. The structure of human ubiquitin in 2-methyl-2,4-pentanediol: a new conformational switch. Protein Sci. 20, 630-639 (2011).

47. Falini, G. et al. Structural probing of $\mathrm{Zn}(\mathrm{II}), \mathrm{Cd}(\mathrm{II})$ and $\mathrm{Hg}(\mathrm{II})$ binding to human ubiquitin. Chem. Commun. 45, 5960-5962 (2008).

48. Kuzmanic, A., Pannu, N. S. \& Zagrovic, B. X-ray refinement significantly underestimates the level of microscopic heterogeneity in biomolecular crystals. Nat. Commun. 5, 3220 (2014).

49. Mollica, L. et al. Atomic-resolution structural dynamics in crystalline proteins from NMR and molecular simulation. J. Phys. Chem. Lett. 3 3657-3662 (2012).

50. Xue, Y. \& Skrynnikov, N. R. Ensemble MD simulations restrained via crystallographic data: accurate structure leads to accurate dynamics. Protein Sci. 23, 488-507 (2014)

51. Stafford, K. A., Robustelli, P. \& Palmer, A. G. Thermal adaptation of conformational dynamics in ribonuclease H. PLoS Comput. Biol. 9, e1003218 (2013).

52. Sidhu, A., Surolia, A., Robertson, A. D. \& Sundd, M. A hydrogen bond regulates slow motions in ubiquitin by modulating a $\beta$-turn flip. J. Mol. Biol. 411, 1037-1048 (2011).

53. Olsson, M. H. M., Sondergaard, C. R., Rostkowski, M. \& Jensen, J. H. PROPKA3: consistent treatment of internal and surface residues in empirical pKa predictions. J. Chem. Theory Comput. 7, 525-537 (2011). 
54. Neal, S., Nip, A. M., Zhang, H. \& Wishart, D. S. Rapid and accurate calculation of protein ${ }^{1} \mathrm{H},{ }^{13} \mathrm{C}$ and ${ }^{15} \mathrm{~N}$ chemical shifts. J. Biomol. NMR 26, 215-240 (2003).

55. Xue, Y., Ward, J. M., Yuwen, T., Podkorytov, I. S. \& Skrynnikov, N. R. Microsecond time-scale conformational exchange in proteins: using long molecular dynamics trajectory to simulate NMR relaxation dispersion data. J. Am. Chem. Soc. 134, 2555-2562 (2012).

56. Glasoe, P., Long, F., McConnell, H. M. \& Redfield, A. G. Use of glass electrodes to measure acidities in deuterium oxide. J. Chem. Phys. 28, 19-31 (1957).

57. Kurauskas, V. et al. Cross-correlated relaxation of dipolar coupling and chemical-shift anisotropy in magic-angle spinning $\mathrm{R}_{1 \rho}$ NMR measurements: application to protein backbone dynamics measurements. J. Phys. Chem. B 120, 8905-8913 (2016).

58. Motulsky, H. \& Christopoulos, A. Fitting Models to Biological Data Using Linear and Nonlinear Regression (GraphPad Software Inc., 2003).

59. Meiboom, S. Nuclear magnetic resonance study of the proton transfer in water. J. Chem. Phys. 34, 375-388 (1961).

60. Morin, S. et al. Relax: the analysis of biomolecular kinetics and thermodynamics using NMR relaxation dispersion data. Bioinformatics 30 2219-2220 (2014).

61. Krushelnitsky, A., Zinkevich, T., Reif, B. \& Saalwächter, K. Slow motions in microcrystalline proteins as observed by MAS-dependent ${ }^{15} \mathrm{~N}$ rotating-frame NMR relaxation. J. Magn. Reson. 248, 8-12 (2014).

62. Hornak, V. et al. Comparison of multiple Amber force fields and development of improved protein backbone parameters. Proteins 65, 712-725 (2006).

63. Best, R. B. \& Hummer, G. Optimized molecular dynamics force fields applied to the helix-coil transition of polypeptides. J. Phys. Chem. B 113, 9004-9015 (2009).

64. Lindorff-Larsen, K. et al. Improved side-chain torsion potentials for the Amber ff99SB protein force field. Proteins Struct. Funct. Bioinf. 78, 1950-1958 (2010).

65. Cerutti, D. S., Le Trong, I., Stenkamp, R. E. \& Lybrand, T. P. Simulations of a protein crystal: explicit treatment of crystallization conditions links theory and experiment in the streptavidin-biotin complex. Biochemistry 47, 12065-12077 (2008).

66. Maier, J. A. et al. ff14SB: improving the accuracy of protein side chain and backbone parameters from ff99SB. J. Chem. Theory Comput. 11, 3696-3713 (2015).

67. Kumar, S. \& Nussinov, R. Relationship between ion pair geometries and electrostatic strengths in proteins. Biophys. J. 83, 1595-1612 (2002).

68. Xue, Y., Yuwen, T., Zhu, F. \& Skrynnikov, N. R. Role of electrostatic interactions in binding of peptides and intrinsically disordered proteins to their folded targets. 1. NMR and MD characterization of the complex between the c-Crk N-SH3 domain and the peptide Sos. Biochemistry 53, 6473-6495 (2014).

69. Han, B., Liu, Y., Ginzinger, S. W. \& Wishart, D. S. SHIFTX2: significantly improved protein chemical shift prediction. J. Biomol. NMR 50, 43-57 (2011).

70. Clore, G. M., Driscoll, P. C., Wingfield, P. T. \& Gronenborn, A. M. Analysis of the backbone dynamics of interleukin-1 beta using two-dimensional inverse detected heteronuclear ${ }^{15} \mathrm{~N}-{ }^{1} \mathrm{H}$ NMR spectroscopy. Biochemistry 29, 7387-7401 (1990).

\section{Acknowledgements}

We thank Troels Schwarz-Linnet (University of Copenhagen) for helpful advice about the use of the program relax for data analysis, as well as Dr. Peixiang Ma, Jens D. Haller Oleg Mikhailovskii, and Manfred Burghammer for insightful discussions. This work was financially supported by the European Research Council (ERC-Stg-2012-311318-ProtDyn2Function), the French Research Agency ANR (ANR 10-PDOC-011-01), as well as Commissariat à l'énérgie atomique et aux énérgies alternatives (CEA), Centre National de la Recherche Scientifique (CNRS) and Université Grenoble Alpes. This work used the platforms of the Grenoble Instruct Center (ISBG; UMS 3518 CNRS-CEA-UJF-EMBL) with support from FRISBI (ANR-10-INSB-05-02) and GRAL (ANR-10-LABX-49-01) within the Grenoble Partnership for Structural Biology (PSB). S.A.I., O.N.R., and N.R.S. acknowledge Russian Science Foundation for financial support of the MD simulation component of this study (RSF grant 15-14-20038). N.C. is supported by a fellowship from the Fondation France Alzheimer.

\section{Author contributions}

V.K. and P.S. performed and analyzed NMR experiments. A.H. and I.A. prepared protein samples and crystals. P.S., A.H., J.W., N.C., J.-P.C., and A.S. crystallized ubiquitin in different crystal forms or characterized crystal samples. S.A.I., O.N.R., Y.X., T.Y., and N.R.S. performed and analyzed MD simulations. P.S. and N.R.S. designed the research. P. S. and N.R.S. wrote the manuscript with contributions from other authors.

\section{Additional information}

Supplementary Information accompanies this paper at doi:10.1038/s41467-017-00165-8.

Competing interest: The authors declare no competing financial interests.

Reprints and permission information is available online at http://npg.nature.com/ reprintsandpermissions/

Publisher's note: Springer Nature remains neutral with regard to jurisdictional claims in published maps and institutional affiliations.

\begin{abstract}
(c) (i) Open Access This article is licensed under a Creative Commons Attribution 4.0 International License, which permits use, sharing, adaptation, distribution and reproduction in any medium or format, as long as you give appropriate credit to the original author(s) and the source, provide a link to the Creative Commons license, and indicate if changes were made. The images or other third party material in this article are included in the article's Creative Commons license, unless indicated otherwise in a credit line to the material. If material is not included in the article's Creative Commons license and your intended use is not permitted by statutory regulation or exceeds the permitted use, you will need to obtain permission directly from the copyright holder. To view a copy of this license, visit http://creativecommons.org/ licenses/by/4.0/.
\end{abstract}

(C) The Author(s) 2017 